\title{
Reversal of intraoperative arterial thrombosis with a fibrinolytic agent when treating large and giant partially thrombosed aneurysms of the middle cerebral artery
}

\author{
Shalva Eliava, MD, PhD, Yury Pilipenko, MD, PhD, Oleg Shekhtman, MD, PhD, and \\ Anton Konovalov, MD
}

Vascular (3rd) Department, Burdenko Neurosurgical Institute, Moscow, Russia

OBJECTIVE Thrombosis of the cerebral arteries is one of the complications of microsurgical operations for partially thrombosed intracranial aneurysms. The object of this study was to assess the frequency of intraoperative arterial thrombosis (IAT) during microsurgical treatment of large and giant partially thrombosed aneurysms of the middle cerebral artery (MCA) and also to assess the efficacy of the treatment of this complication.

MATERIALS The authors analyzed a consecutive series of 53 patients who underwent surgery for partially thrombosed aneurysms of the MCA at the Burdenko Neurosurgical Institute between January 2005 and September 2014. Thirty-two patients had large aneurysms $(15-25 \mathrm{~mm})$ and 21 had giant aneurysms $(>25 \mathrm{~mm})$. Clipping of aneurysms was performed in 47 patients, trapping was performed in 3 , and wrapping was performed in 3 .

RESULTS IAT was diagnosed in 10 patients (18.9\%). The authors describe a technique for IAT reversal involving the injection of modified human recombinant prourokinase (mr-proUK). Of the 7 patients who underwent injection of mr-proUK in the thrombosed artery, $5(71.4 \%)$ were discharged without any change in neurological status, $1(14.3 \%)$ experienced moderate deterioration (modified Rankin Scale [mRS] score of 2), and $1(14.3 \%)$ experienced severe deterioration (mRS score of 4). Among the 3 patients who had complications and did not receive an injection of mr-proUK, 2 experienced severe deterioration (mRS score of 3 and 4 ) and 1 had moderate deterioration (mRS score of 2).

CONCLUSIONS The primary risk factor of thrombectomy with temporary trapping is thrombosis in the MCA branches. The injection of a fibrinolytic agent into thrombosed MCA branches is an effective method for the treatment of IAT. http://thejns.org/doi/abs/10.3171/2015.2.JNS142655

KEY WORDS partially thrombosed aneurysm; large aneurysm; giant aneurysm; thrombectomy; cerebral artery thrombosis; fibrinolysis; prourokinase; MCA aneurysm; vascular disorders

$\Lambda$ PPROXIMATELY $40 \%$ to $50 \%$ of large and giant cerebral aneurysms contain an intraluminal thrombus. ${ }^{2,8,12}$ The risk of rupture of partially thrombosed aneurysms is comparable to the risk of rupture of nonthrombosed aneurysms; additionally, the probability of ischemic stroke due to distal thromboembolism is high. ${ }^{2,8,22}$ In this regard, patients harboring aneurysms with an intraluminal thrombus are candidates for surgical treatment. $2,8,11,17$

The Burdenko Neurosurgical Institute has extensive experience in the treatment of large and giant partially thrombosed aneurysms of the middle cerebral artery (MCA). At this institute, the vast majority of these aneurysms have been treated using microsurgical techniques. Thrombosis of the branches of the MCA is one of the most serious complications of microsurgery and can cause severe neurological deficits.

The object of this study was to assess the frequency of intraoperative arterial thrombosis (IAT) during microsurgical treatment of large and giant partially thrombosed

ABBREVIATIONS CTA = CT angiography; DSA = digital subtraction angiography; IAT = intraoperative arterial thrombosis; $M C A=$ middle cerebral artery; mr-proUK = modified human recombinant prourokinase; $\mathrm{mRS}=$ modified Rankin Scale.

SUBMITTED December 2, 2014. ACCEPTED February 25, 2015.

INCLUDE WHEN CITING Published online October 2, 2015; DOI: 10.3171/2015.2.JNS142655. 
aneurysms of the MCA and the efficacy of treatment of the complication.

\section{Methods}

\section{Characteristics of the Study Group}

The study group included 53 patients who underwent microsurgical treatment for large and giant partially thrombosed MCA aneurysms in the Burdenko Neurosurgical Institute from January 2005 to September 2014.

The mean age of patients was 43.6 years (range 26-67 years). Twenty-nine of the patients were male, and 24 were female. Forty patients had histories of hemorrhage, and 13 did not. Thirty-two patients presented with large aneurysms (15-25 $\mathrm{mm}$ ), and 21 presented with giant aneurysms $(>25 \mathrm{~mm})$. Patients with acute-stage subarachnoid hemorrhage operated on within 2 weeks were not included. Treatment risks in patients with acute hemorrhages are associated with other factors (e.g., vasospasm, edema, and hydrocephalus). In general, thromboses in such aneurysms are not organized and have soft structures that are not a serious complicating surgical factor.

The sizes and types of aneurysms were studied with contrast-enhanced CT scanning, MRI, CT angiography (CTA), or digital subtraction angiography (DSA). An aneurysm was considered partially thrombosed when the area of contrast enhancement was smaller than the overall aneurysm size.

All MCA aneurysms with intraluminal thrombi were divided into 3 types: Type I, partially thrombosed saccular aneurysms with extension of the thrombus into the neck (13 patients); Type II, partially thrombosed saccular aneurysms containing a thrombus in the dome or fundus (i.e., the thrombus did not extend to or involve the aneurysm neck; 32 patients); Type III, partially thrombosed fusiform aneurysms (8 patients).

\section{Treatment}

All patients were treated with microsurgery. Vessel patency during surgery was assessed with a micro-Doppler ultrasound device (DWL MultiDop P, 16 MHz; Compumedics).

The general characteristics of the surgical procedures and intra- and postoperative complications by aneurysm type are listed in Table 1.

\section{Neurological Complications}

The severity of the neurological complications were classified as follows: moderate deterioration, severe deterioration, or death. Moderate deterioration was defined as new or worsened neurological symptoms without mental, speech, or motor impairment. Severe deterioration included new or worsened mental, speech, motor, or bulbar disorders.

The severity of the neurological disorders in the patients who underwent surgery was also evaluated using the modified Rankin Scale (mRS). The evaluation of neurological status was performed 1-3 weeks following surgery, depending on the patient's condition.
TABLE 1. Characteristics of partially thrombosed MCA aneurysm subgroups*

\begin{tabular}{lrrcc}
\hline \multicolumn{1}{c}{ Characteristic } & $\begin{array}{c}\text { Type I } \\
(\mathrm{n}=13)\end{array}$ & $\begin{array}{c}\text { Type II } \\
(\mathrm{n}=32)\end{array}$ & $\begin{array}{c}\text { Type III } \\
(\mathrm{n}=8)\end{array}$ & $\begin{array}{c}\text { Total } \\
(\mathrm{n}=53)\end{array}$ \\
\hline Aneurysm size & & & & \\
\hline$\quad$ Large & 5 & 23 & 4 & 32 \\
\hline$\quad$ Giant & 8 & 9 & 4 & 21 \\
\hline Treatment & 11 & 32 & 4 & 47 \\
\hline$\quad$ Clipping & 0 & 0 & 3 & 3 \\
\hline$\quad$ Trapping & 2 & 0 & 1 & 3 \\
\hline Wrapping & & & & \\
\hline Complications & 0 & 5 & 0 & 5 \\
\hline$\quad$ Intraop aneurysm rupture & 6 & 1 & 3 & 10 \\
\hline$\quad$ IAT & & & & \\
\hline Outcomes & 1 & 1 & 1 & 3 \\
\hline$\quad$ Moderate deterioration & 2 & 2 & 2 & 6 \\
\hline$\quad$ Severe deterioration & 0 & 1 & 0 & 1 \\
\hline$\quad$ Death & & & & \\
\hline
\end{tabular}

* See Results for definitions of the aneurysm types. Values represent the number of patients.

\section{Results}

The choice of surgical treatment depended on the type of the partially thrombosed aneurysm; therefore, the outcomes are given separately for each group.

\section{Type I}

These 13 patients had partially thrombosed saccular aneurysms with extension of the thrombus into the neck (Type I). Conventional aneurysm clipping was not possible in this group because heavy thrombotic masses prevented the clips from completely closing.

In 11 of the 13 cases, the aneurysm neck was clipped after thrombectomy. In 2 patients with unruptured aneurysms, clipping was not attempted because of the presence of intraluminal thrombus and severe atherosclerosis of the parent arteries and aneurysm neck, which were observed at surgery. In both cases, the aneurysm walls were wrapped with surgical gauze and cyanoacrylate glue (Omnex, Ethicon).

In all cases, thrombectomies were performed with temporary trapping. The mean trapping time for the thrombectomies was 10 minutes (range 4-20 minutes).

Aneurysmotomy was performed to provide direct access to the thrombus in the neck while preserving sufficient margins for clipping. Initially, heavily organized thrombotic masses in the central part of the aneurysm were destroyed using a Cavitron ultrasonic aspirator (Integra) or gouge forceps to increase the intraluminal volume. Then, the thrombi directly adjacent to the neck were removed with a sharp dissector and forceps. Before clipping, the aneurysm lumen was washed with saline. Clipping of the aneurysm neck was performed with a single clip or a combination of several clips of various configurations while preserving the lumina of the MCA and its branches.

Major intraoperative complications after clipping of 
the neck of the aneurysm included thrombotic occlusion of the secondary MCA branches (6 cases). In all cases, the presence of thrombi was confirmed with microvascular Doppler ultrasound probes that demonstrated occlusion ranging from progressive slowing of linear blood flow velocity in the $\mathrm{M}_{2}$ branches for 1-2 minutes to complete blockage. After temporary clipping of the $\mathrm{M}_{1}$ segment and removal of the neck clips, we noted minimal bleeding, or none, from the thrombosed MCA branches. Attempts at direct reversal of acute thrombus through the open dome of the aneurysm and irrigation of the branch with saline plus heparin (5000 IU of heparin per $20 \mathrm{ml}$ of saline) led to Doppler ultrasound-detected restoration of blood flow in one of the first patients (Table 2, Case 1) in whom this complication occurred. Subsequently these measures were found to be insufficient because the postoperative period was complicated by ischemic damage to the thrombosed artery.

In the next 5 cases, we used a fibrinolytic agent, modified human recombinant prourokinase (mr-proUK), to resolve the intraoperatively diagnosed arterial thromboses.

We used an insulin needle to puncture the proximal thrombosed vessel or aneurysm neck in the area of the artery ostium and injected mr-proUK intraarterially (Fig. 1). Pinpoint bleeding following the puncture was stopped with a cottonoid applied for 1-2 minutes. Restoration of blood flow was noted at 1-3 minutes after mr-proUK administration. When one of the two branches of the MCA bifurcation was thrombosed, we applied a temporary clip to the $\mathrm{M}_{1}$ segment prior to puncture to prevent migration of the fibrinolytic agent with blood flow into the second functioning branch.

We managed to restore blood flow and avoid severe ischemic damage in 3 of the 5 patients in this group who were treated with mr-proUK. In 1 patient (Table 2, Case 4), due to the lack of effect of multiple fractional injections of the fibrinolytic agent into the thrombosed $\mathrm{M}_{2}$ branch, we performed distal revascularization via a bypass with the superficial temporal artery, which resulted in a good clinical outcome (Fig. 2). Another patient in whom both of the secondary MCA branches were thrombosed (Table 2, Case 2) also underwent repeated puncture and intraarterial mr-proUK injection into the MCA bifurcation and directly into the thrombosed vessels, but only partial blood flow restoration was achieved. Microvascular Doppler ultrasound revealed that blood flow in the 2 thrombosed branches was reduced two- to threefold in comparison with baseline. Postoperatively, a large area of ischemia with dislocation syndrome developed and required decompressive craniectomy (Fig. 3).

No postoperative hemorrhagic complications following intraarterial mr-proUK administration were recorded.

Thus, IAT occurred in $6(54.5 \%)$ of 11 patients who underwent thrombectomy at the aneurysm neck. Angiography confirmed the micro-Doppler ultrasound data regarding blood flow restoration in the intraoperatively thrombosed MCA branches in 3 of the 5 patients who received mr-proUK.

The neurological outcomes of surgery in the 13 patients with partially thrombosed aneurysms (thrombus extension to the neck) were as follows: no change (mRS Score $0-2)$ in 10 patients (76.9\%), moderate deterioration (mRS Score 3) in 1 patient (7.7\%), and severe deterioration (mRS Score 4) in 2 patients (15.4\%) with IAT (Table 2, Cases 1 and 2). No deaths were recorded in this group.

\section{Type II}

This group consisted of 32 patients with partially thrombosed saccular aneurysms without thrombus in the neck (Type II).

All 32 patients in this group underwent clipping of the neck of the aneurysm (Fig. 4); 21 patients underwent temporary clipping of the $\mathrm{M}_{1}$ segment, 3 underwent temporary trapping (one $\mathrm{M}_{1}$ and two $\mathrm{M}_{2}$ segments), and 8 did not have temporary clipping.

In 10 cases (31.3\%) in this group, stenosis of one of the MCA branches was revealed after clipping because of the thrombus in the dome ramping the clips and causing

TABLE 2. Patients with intraoperative thromboses of the MCA branches

\begin{tabular}{|c|c|c|c|c|c|c|c|c|c|c|}
\hline $\begin{array}{l}\text { Lesion Type } \\
\& \text { Case No. }\end{array}$ & Sex & $\begin{array}{l}\text { Age } \\
\text { (yrs) }\end{array}$ & $\begin{array}{c}\text { Yr of } \\
\text { Tx }\end{array}$ & $\begin{array}{c}\text { Location of } \\
\text { Aneurysm }\end{array}$ & $\begin{array}{c}\text { Size of } \\
\text { Aneurysm }\end{array}$ & Surgery & Fibrinolysis & Ischemia & Neurological Status & $\begin{array}{l}\mathrm{mRS} \\
\text { Score }\end{array}$ \\
\hline \multicolumn{11}{|c|}{ Type I: partially thrombosed saccular aneurysm w/ thrombus in the neck } \\
\hline 1 & M & 39 & 2006 & MCA bif & Giant & ANC & No & Temporal lobe & Severe deterioration & 3 \\
\hline 2 & M & 56 & 2011 & MCA bif & Giant & ANC & Yes & Rt hemisphere, dislocation & Severe deterioration & 4 \\
\hline 3 & M & 43 & 2011 & MCA bif & Large & ANC & Yes & No & Unchanged & 2 \\
\hline 4 & $\mathrm{M}$ & 56 & 2013 & MCA bif & Giant & ANC + bypass & Yes & Frontal lobe & Moderate deterioration & 2 \\
\hline 5 & M & 62 & 2014 & MCA bif & Giant & ANC & Yes & No & Unchanged & 1 \\
\hline 6 & M & 30 & 2014 & MCA bif & Giant & ANC & Yes & No & Unchanged & 0 \\
\hline \multicolumn{11}{|c|}{ Type II: partially thrombosed saccular aneurysm w/o thrombus in the neck } \\
\hline 7 & $\mathrm{~F}$ & 54 & 2013 & MCA bif & Large & ANC & Yes & No & Unchanged & 0 \\
\hline \multicolumn{11}{|c|}{ Type III: partially thrombosed fusiform aneurysm } \\
\hline 8 & M & 44 & 2007 & $\mathrm{MCA} \mathrm{M}_{1}$ & Giant & CLR & No & No (hematoma) & Severe deterioration & 4 \\
\hline 9 & $\mathrm{~F}$ & 26 & 2010 & $\mathrm{MCA} \mathrm{M}_{2}$ & Giant & CLR & Yes & No & Moderate deterioration & 2 \\
\hline 10 & $\mathrm{~F}$ & 52 & 2012 & $\mathrm{MCA} \mathrm{M}_{3}$ & Large & Bypass & Yes & No & Unchanged & 1 \\
\hline
\end{tabular}

ANC = aneurysm neck clipping; bif = bifurcation; $C L R=$ clipping with artery lumen reconstruction; $T x=$ treatment. 


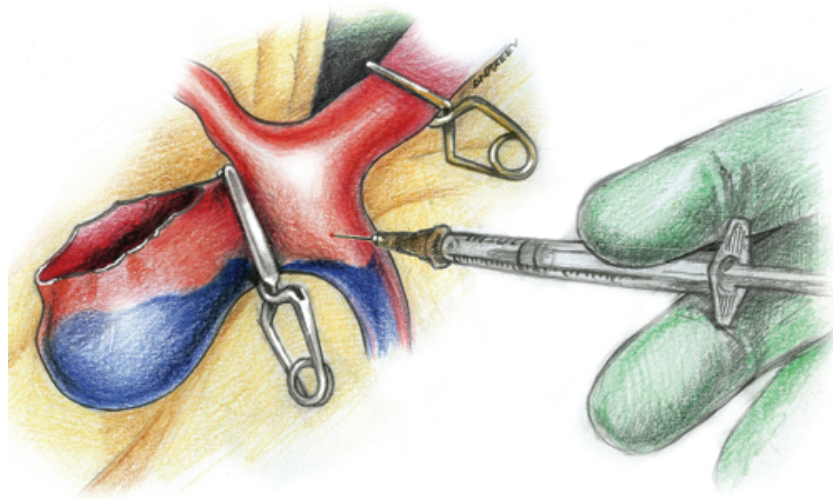

FIG. 1. Illustration showing treatment of thrombosis in the branch artery. mr-proUK is injected into the thrombosed artery. Copyright Dmitry Andreev. Published with permission. Figure is available in color online only.

them to slide down onto the parent artery. This situation required thrombectomy at the aneurysm dome and distal clip repositioning.

Major intraoperative complications during neck dissection and clipping included aneurysm rupture (5 cases, $15.6 \%)$. Two of these patients experienced postoperative complications. One 52-year-old woman developed leftsided hemiparesis in the postoperative period due to damage to the distal lenticulostriate artery. Another 41-yearold female patient with a giant MCA aneurysm sustained extended aneurysm rupture involving the neck of the aneurysm during surgery. CT revealed a large ischemic area in the right parietal-temporal region due to the prolonged clipping (30 minutes) and anemia. The patient died of septic complications while in the ICU.

Notably, all 5 of the patients with complications developed ruptures in the area between thickened and thin parts of the aneurysm wall, and 3 patients required thrombectomy at the site adjacent to the rupture to place the clip and stop the bleeding.

Only $1(3.1 \%)$ of the 32 patients in this group, a 54-yearold woman, developed IAT (Table 2, Case 7). After temporary trapping and clipping of the neck of the large aneurysm, the patient developed Doppler ultrasound-detected progressive decline in linear blood flow velocity in one of the MCA trifurcation branches with visible arterial darkening and a lack of pulsation. Revision of the artery ostium revealed no significant stenosis due to the clip. One minute after puncture of the thrombosed artery and the intraarterial injection of 50,000 IU mr-proUK, Doppler ultrasound indicated restoration of blood flow and pulsation and a correction of the artery discoloration. No postoperative complications were observed.

To confirm the lack of blood flow in the aneurysm lumina after neck clipping, 27 patients underwent aneurysmotomies in thrombus-free areas. In 5 of these cases, follow-up angiography revealed complete angiographic obliteration.

In general, the neurological outcomes of surgical treatment in the 32 patients with partially thrombosed MCA aneurysms without thrombi in the neck area were as follows: no change (mRS Score $0-2)$ in 28 patients $(87.5 \%)$, moderate deterioration (mRS Score 2 ) in 1 patient $(3.1 \%)$,
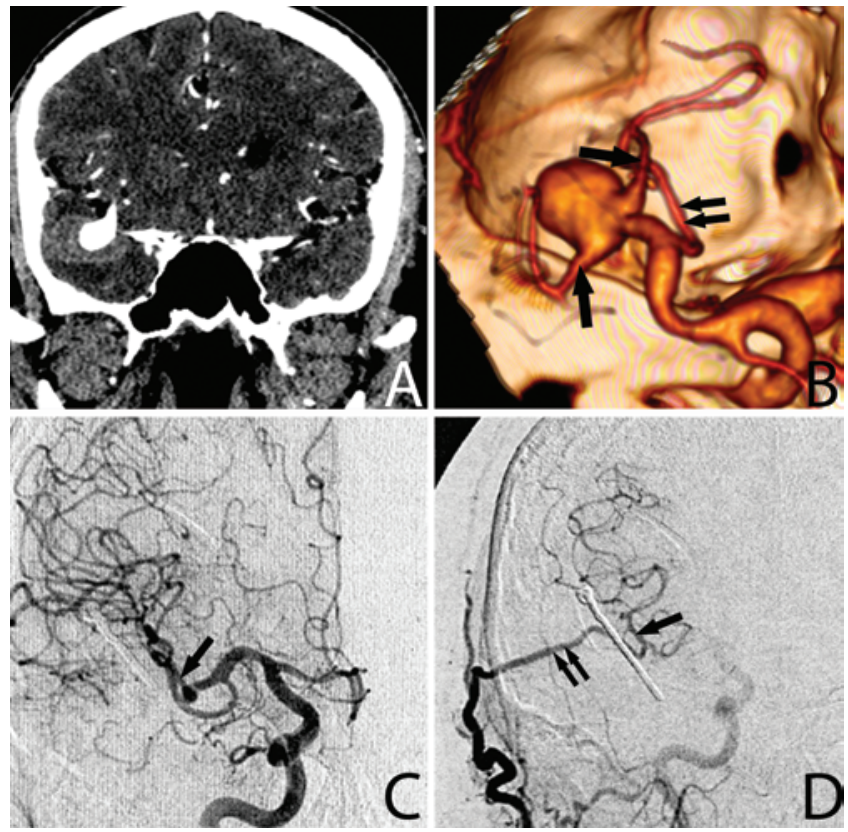

FIG. 2. Case 4. Bypass was performed between the superficial temporal artery and the thrombosed $\mathrm{M}_{2}$ segment of the right MCA after thrombectomy and clipping of the aneurysm neck. A: Preoperative CT scan shows part of the thrombosed aneurysm. B: Preoperative 3D CT reconstruction shows blood flow through the neck of the aneurysm. Single arrows indicate $\mathrm{M}_{2}$ branches, and double arrows indicate the temporal branch of the MCA. C: Postoperative DSA image demonstrates contrast in the right internal carotid artery system. Arrow indicates the preserved $\mathrm{M}_{2}$ branch. D: Postoperative DSA image shows contrast in the right external carotid artery system. Single arrow indicates the revascularized $\mathrm{M}_{2}$ branches, and double arrows indicate the temporal branch of the MCA (donor). Figure is available in color online only.

severe deterioration (mRS Score 3-4) in 2 patients (6.3\%), and death in 1 patient (3.1\%).

\section{Type III}

This group consisted of patients with partially thrombosed fusiform aneurysms (Type III). None of the 8 patients in this group was amenable to conventional clipping.

Two patients had giant aneurysms in the $\mathrm{M}_{1}$ segment of the MCA. Preoperative imaging revealed thrombi in one of the walls (eccentric) rather than completely circumferential thrombi in the aneurysm lumen (concentric). The surgery plan included debulking of the dome with temporary trapping and clipping of the eccentric portion to achieve reconstruction of the $\mathrm{M}_{1}$ lumen.

A 44-year-old patient developed a thrombus in one branch of the left MCA bifurcation intraoperatively due to the procedure (trapping time 6 minutes, 45 seconds; Table 2, Case 8). Direct thrombectomy at the ostium of the $\mathrm{M}_{2}$ segment was performed and the MCA branches were irrigated with heparinized normal saline to allow for the reconstruction of the lumen of the thrombosed $\mathrm{M}_{2}$ segment. In the early postoperative period, CT revealed a large intracerebral hematoma that required reoperation for evacuation. Neurologically, the patient exhibited severe postoperative right-sided hemiparesis and aphasia.

A 27-year-old female patient also underwent thrombec- 


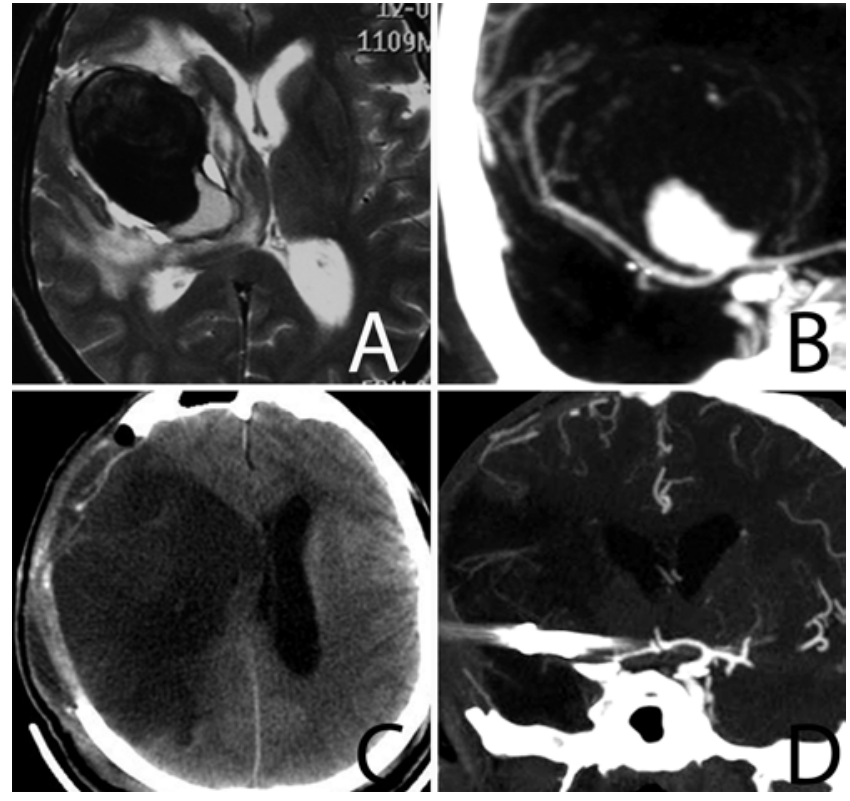

FIG. 3. Case 2. Large ischemic area in right hemisphere due to IAT of the 2 MCA branches after thrombectomy and clipping of a giant aneurysm. A: Preoperative MR image shows thrombosed part of the aneurysm. B: Preoperative CTA image shows functioning part of the aneurysm. C: CT scan obtained 1 week after surgery shows ischemia of the right hemisphere. D: CTA image acquired 3 weeks after surgery shows no contrast in the right MCA.

tomy with temporary trapping (5 minutes) and clipping of the eccentric giant aneurysm to reconstruct the lumen of the $\mathrm{M}_{1}$ segment of the right MCA. No intraoperative complications were observed. In the postoperative period, severe left-sided hemiparesis developed, and CT revealed a small ischemic area in the right subcortical nuclei that was associated with a lenticulostriate artery infarct. This patient received conservative treatment only.

One 57-year-old female patient underwent surgery for a fusiform, partially thrombosed aneurysm of the left MCA bifurcation. During this surgery, significant atherosclerotic plaques were found in the aneurysm walls and the distal secondary MCA branches, in addition to a thrombus in the aneurysm dome. We decided not to attempt aneurysm exclusion; instead, the walls were wrapped with surgical gauze and Omnex glue.

In 5 cases partially thrombosed fusiform aneurysms of the distal MCA branches were diagnosed. Two patients underwent aneurysm clipping with luminal reconstruction of the $\mathrm{M}_{2}$ segment after thrombectomy with temporary trapping. One of these patients (Table 2, Case 9) developed distal artery thrombosis after clipping. After direct removal of the soft thrombus from the proximal $\mathrm{M}_{3}$ segment and intraarterial injection of 25,000 IU urokinase, the artery lumen was reconstructed. Moderate speech disorders developed but significantly diminished within 7 days postoperatively.

In 3 patients, nearly completely thrombosed fusiform aneurysms were diagnosed. We decided not to attempt clipping with reconstruction of the parent artery. In 1 patient with an aneurysm in the $\mathrm{M}_{4}$ segment of the MCA, we performed aneurysm trapping. In the second case, the aneurysm was located in the $\mathrm{M}_{2}$ segment, and trapping was performed after creation of a bypass between the temporal MCA branch and the $\mathrm{M}_{3}$ segment (Fig. 5). In the other patient (Table 2, Case 10), a large aneurysm of the $\mathrm{M}_{3}$ segment was excised following end-to-end (in situ) bypass.

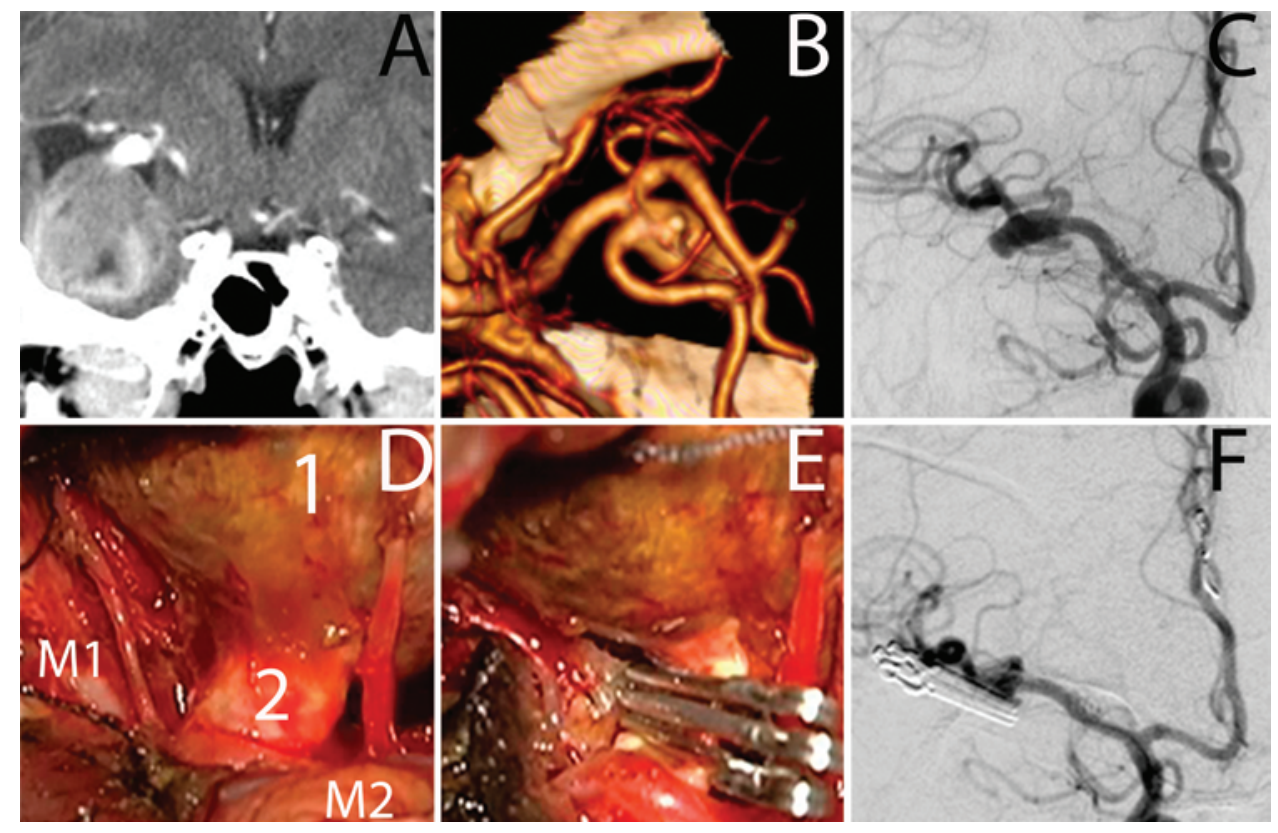

FIG. 4. Clipping of the neck of an almost completely thrombosed giant aneurysm in the right MCA bifurcation in a 54-year-old woman. A: Preoperative CT scan shows thrombosis in aneurysm dome. B and C: Preoperative CTA image and 3D CT reconstruction show blood flowing only through the aneurysm neck. D and E: Intraoperative photographs show thrombi in the dome (labeled 1) and neck (labeled 2) of the aneurysm. F: Postoperative DSA image shows complete exclusion of aneurysm. Figure is available in color online only. 

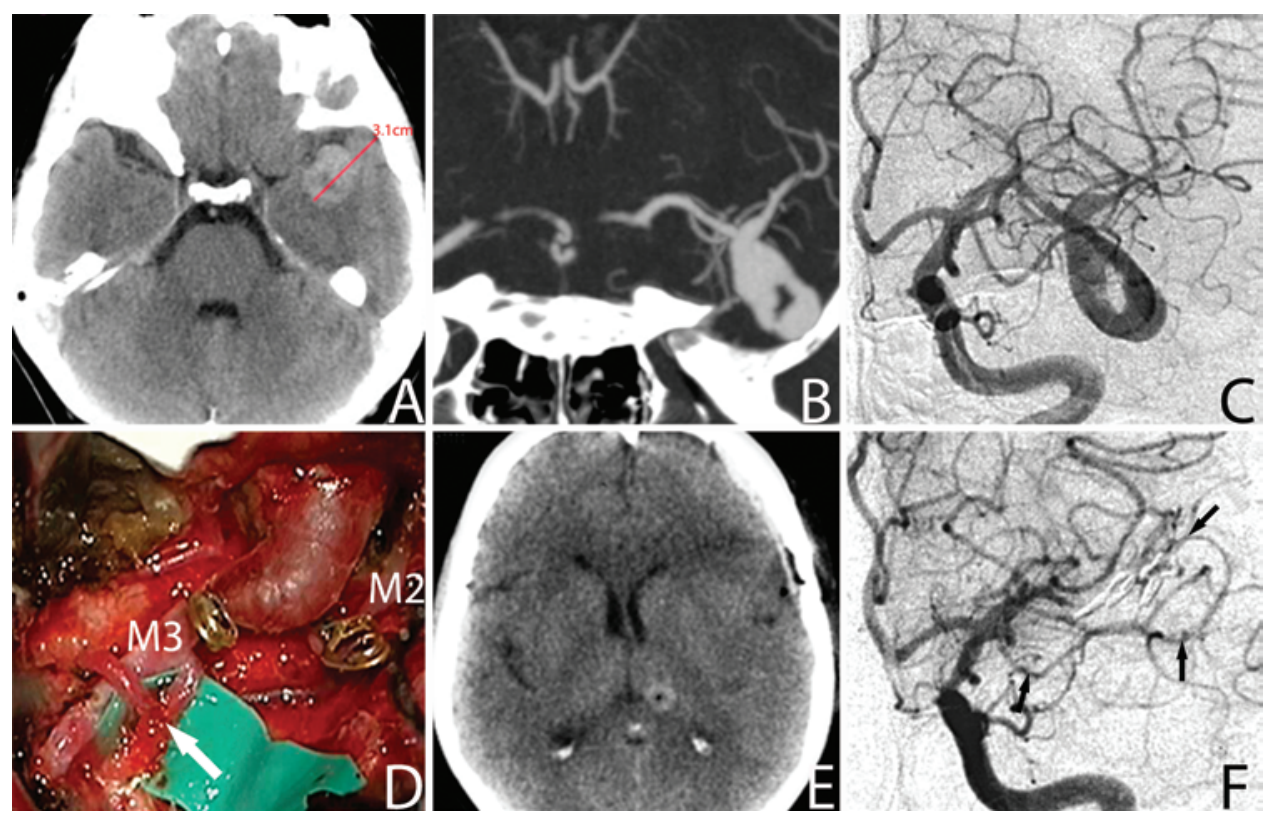

FIG. 5. Trapping of a partially thrombosed fusiform aneurysm in the $M_{2}$ segment of the left MCA after creation of a bypass between the temporal branch and the $\mathrm{M}_{3}$ segment of the left MCA. A: Preoperative CT scan shows thrombosed aneurysm dome. B and C: Preoperative CTA and DSA images show fusiform (looplike) aneurysm. D: Intraoperative photograph shows aneurysm trapping after the creation of an end-to-side bypass (arrow). E: Postoperative CT scan shows no ischemic damage. F: Postoperative DSA image shows complete aneurysm exclusion. The left temporal MCA, the "donor" of the distal portions of $\mathrm{M}_{2}$ segment that had been excluded with the aneurysm, is indicated by arrows. Figure is available in color online only.

Due to significant tension and spasm, a thrombus formed in the area of the bypass. After injection of 50,000 IU mrproUK into the proximal part of the thrombosed vessel, blood flow was restored in 1 minute.

In the preceding 3 cases, no postoperative complications were observed.

Of the 8 patients with partially thrombosed fusiform MCA aneurysms, neurological status remained unchanged in $5(62.5 \%)$ patients. Moderate deterioration (mRS Score 2) was observed in $1(12.5 \%)$ patient, and severe deterioration (mRS Score 3 and $\mathrm{mRS}$ Score 4) was observed in $2(25 \%)$ patients with aneurysms of the $M_{1}$ segment. No deaths were recorded.

Postoperative angiography was performed in 6 patients. A subtotal clipping of the fusiform aneurysm was noted in only 1 patient who had undergone clipping of an $\mathbf{M}_{1}$ segment aneurysm with MCA lumen reconstruction.

\section{Analysis of Outcomes in Patients With IAT}

In all of these patients, the aneurysms had been excluded with temporary trapping. Overall, temporary trapping was performed in 21 patients, and IAT developed in $47.6 \%$ of the cases in which this technique was applied.

Chronologically, in the first 2 patients (treated in 20062007), irrigation of the thrombosed branches with normal saline plus heparin was used to reverse the IAT. Postoperative complications developed in both cases (Table 2, Cases 1 and 8$)$.

In the third IAT case, which presented in 2010 (Table 2, Case 9), an intraarterial injection of urokinase was performed through the dome of the opened aneurysm; despite restoration of blood flow in the thrombosed left MCA branch, moderate speech disorders were observed after surgery.

Since 2011, we have used intraarterially administered mr-proUK to resolve intraoperative thromboses of the MCA branches.

The dose of mr-proUK was selected empirically. In the first 5 cases (Table 2, Cases 2-4, 7, and 10) the applied doses were 35,000-50,000 IU. In 2 of these patients (Table 2 , Cases 2 and 4), no adequate restoration of blood flow in the thrombosed branches occurred, which resulted in postoperative ischemic lesions. In Cases 5 and 6, the doses were 100,000 IU; in both of these patients, blood flow through the thrombosed $\mathrm{M}_{2}$ branches was restored without postoperative complications (Fig. 6).

Overall, among the 7 patients with IAT who underwent intraarterial fibrinolysis with mr-proUK, 5 (71.4\%) were discharged with unchanged neurological status, 1 (14.3\%) patient exhibited moderate deterioration (mRS score 2), and $1(14.3 \%)$ exhibited severe deterioration (mRS score 4).

\section{Discussion}

According to the experience at the Burdenko Neurosurgical Institute and the published data, ${ }^{6,16,17,19}$ microsurgery is the primary method of treatment for large and giant partially thrombosed MCA aneurysms.

To determine the treatment strategy for cases of partially thrombosed saccular MCA aneurysms, one should first focus on the absence or presence of a thrombus in the neck. ${ }^{11,17,21}$ According to this criterion, we identified 2 types of MCA aneurysms. For the first type, we performed conventional neck clipping, and for the second, we performed neck clipping after preliminary thrombectomy. 

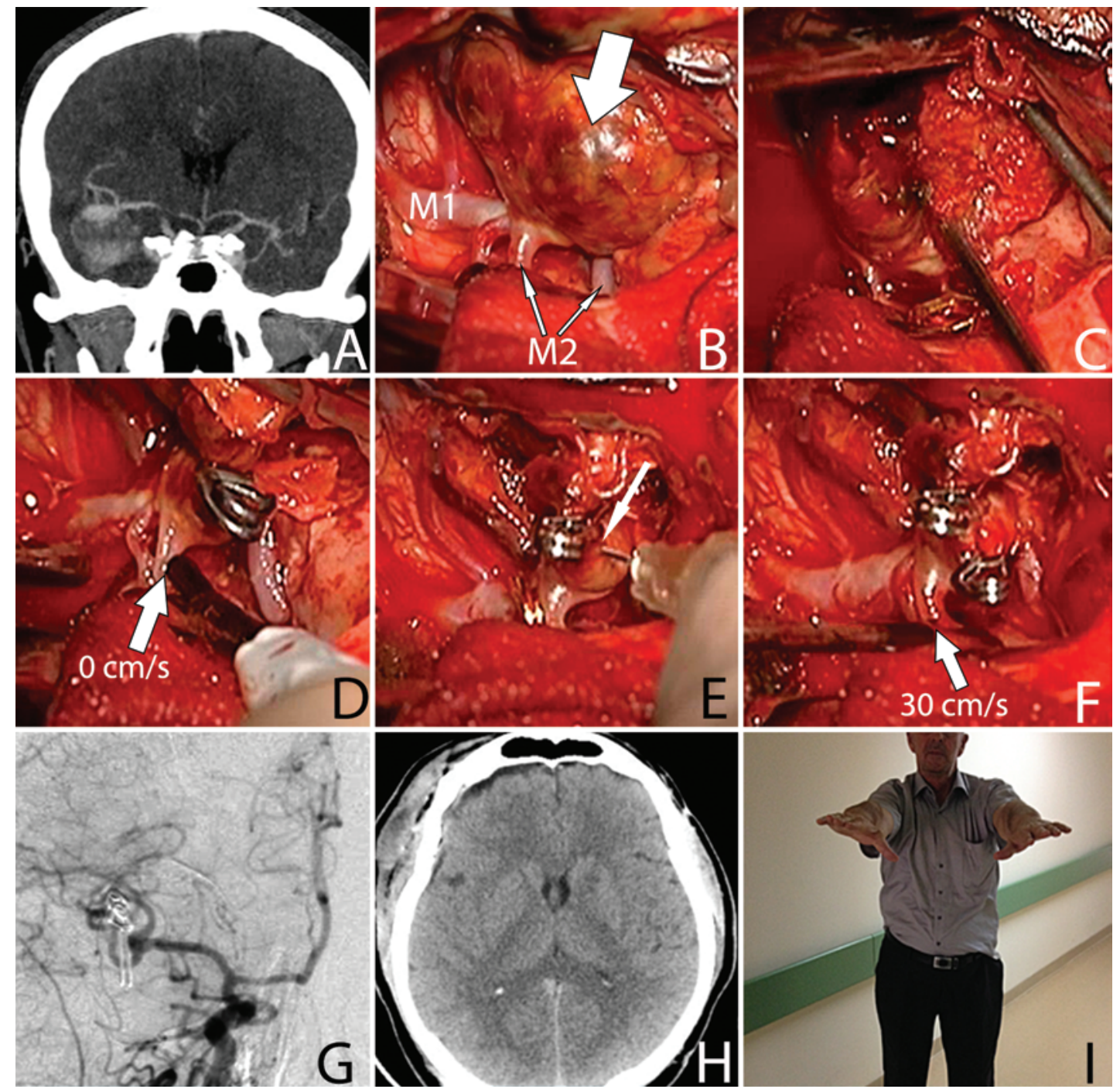

FIG. 6. Case 5. Injection of mr-proUK into the thrombosed right MCA $M_{2}$ segment in a 62-year-old patient. A: Preoperative CTA scan. B-F: Intraoperative photographs. B: Thrombosed aneurysm dome is indicated with arrow. C: Thrombectomy. D: Clipping of the neck. Micro-Doppler ultrasound (arrow) revealed a lack of blood flow in the $\mathrm{M}_{2}$ branch of the MCA. E: Puncture of the functioning neck (arrow) and injection of the fibrinolytic agent. F: Restoration of blood flow in the $\mathrm{M}_{2}$ segment of MCA $(30 \mathrm{~cm} / \mathrm{sec})$ is confirmed by micro-Doppler ultrasound (arrow). G: Postoperative DSA. H: Postoperative CT scan. I: Photograph of the patient after recovery from surgery. Figure is available in color online only.

In cases of partially thrombosed fusiform MCA aneurysms, the surgical strategy is determined by the aneurysm location, thrombus size, and wall thickness. ${ }^{11,17,19}$ We believe that thrombectomy and clipping with reconstruction of the artery lumen are possible only when the thrombus is located primarily on one side of the fusiform aneurysm. In our group, the majority of aneurysms in the $\mathrm{M}_{2}$ segment fulfilled this criterion. Such surgery for fusiform aneurysms of the $M_{1}$ segment caused severe postoperative neurological disorders in 2 cases.

From our perspective, blood flow through fusiform aneurysms with completely circumferential thrombi is scant. Safe thrombectomies for reconstructive clipping are virtually impossible, and bypass is the only option for microsurgical treatment. ${ }^{11,17,19}$

The final decision regarding the feasibility of clipping of any type of partially thrombosed aneurysm can only be made during the surgery because preoperative prediction of the extent of atherosclerotic aneurysm damage is difficult. ${ }^{11,17,21}$

The short-term postoperative results indicated that the maximum number $(87.5 \%)$ of good functional outcomes was achieved in patients with partially thrombosed MCA aneurysms in whom the thrombus was not located in the neck of the aneurysm.

Good results were obtained in $76.9 \%$ of the patients with saccular aneurysms and thrombus extension to the neck and in $62.5 \%$ of the patients with partially thrombosed fusiform aneurysms. A few additional complications in these groups were associated with the increased complexity of surgery due to the need for aneurysmotomy and thrombectomy. 
Thrombosis of one or more MCA branches was the major intraoperative complication of clipping after thrombectomy in our patients.

IAT following clipping of the aneurysm neck should be differentiated from external artery compression with a clip due to improper placing or occlusion by an atherosclerotic plaque. External compression can be eliminated by placing additional distal clips and subsequently removing the original clip.

In addition to embolism, the possible mechanisms of arterial thrombosis following thrombectomy with temporary trapping include increased clotting due to the release of coagulation factors from the thrombus and the atherosclerotic plaques, anatomical narrowing, and vasospasm. The possibility cannot be excluded that altered arterial endothelium in the areas where temporary clips are applied ${ }^{3,9,10}$ might serve as sites for acute thrombus adhesion.

Ischemic complications after surgery for partially thrombosed aneurysms are typically diagnosed in the early postoperative period..$^{10,14,21}$ It is apparent that timely resolution of IAT can be achieved by methods that include administration of intraarterial fibrinolytic agents. Currently, we use mr-proUK in the Burdenko Neurosurgical Institute to treat IATs that develop during surgery for large and giant partially thrombosed MCA aneurysms. In contrast to urokinase, mr-proUK is a selective fibrinolytic that predominantly affects acute thrombi without systemic plasminogen activation and antiplasmin depletion, which significantly reduces the risk of hemorrhagic complications. ${ }^{1,7,13}$

Although it has not been approved for use in the US, we have administered mr-proUK intravenously for the treatment of thrombotic occlusions of coronary and pulmonary arteries. ${ }^{5,20}$ Intraarterially administered mr-proUK has also been used successfully in the treatment of MCA thrombosis, ${ }^{4}$ and the fibrinolytic agent has been locally administered through a catheter to drain intracerebral hematomas. ${ }^{7,15,18}$ Even though mr-proUK has not been approved for use in the US, we assume that tissue plasminogen activator could be used in a similar manner.

In our patients with IAT, we decided to inject $\mathrm{mr}$ proUK into the thrombosed vessel via puncture with a fine needle during microsurgery rather than using the typical endovascular selective fibrinolytic infusion.

In 5 (71.4\%) of the 7 patients in whom mr-proUK was injected into the thrombosed MCA branch during surgery, it was possible to reconstruct the arterial lumen and avoid neurological complications. In the first cases, the failure of this method might have been associated with the lower dose of mr-proUK that was administered. Presently, we believe that the optimal dose for a single-injection administration is $100,000 \mathrm{IU}$ of mr-proUK diluted in 1-2 ml of normal saline.

When the treatment protocol includes thrombectomy, it is important to prevent IAT by attempting to shorten the time required for trapping and removing thrombus and for washing the aneurysm lumen before clipping. In recent years, we have alternately opened the jaws of the distal temporary clips before clipping to eliminate possible MCA branch occlusion by thrombus fragments and atherosclerotic plaques after thrombectomy. Bleeding due to retrograde flow is typically mild, and the occluding fragments can be washed out of the MCA branches.
Some authors have proposed that thrombectomy with trapping should be abandoned in favor of revascularization procedures due to the high risk of complications and mortality. ${ }^{11,17}$ Our experience with microsurgical treatment of large and giant partially thrombosed MCA aneurysms revealed the possibility of resolving intraoperative problems associated with arterial thrombosis after thrombectomy and therefore avoiding deaths and achieving good outcomes in most cases.

The following is a proposed algorithm that can be applied during surgery in cases of lack of blood flow in one or more MCA branches after trapping, thrombectomy, and aneurysm neck clipping: 1) Rule out external compression of the dysfunctional artery by the clip and, if necessary, reposition the clip. 2) Apply cottonoid with papaverine to the dysfunctional artery and, after 1-2 minutes, assess blood flow with micro-Doppler ultrasound or fluorescent angiography. 3) Puncture the proximal parts of the thrombosed vessel with an insulin needle and inject a fibrinolytic (100,000 IU mr-proUK). Press the puncture site with a cottonoid and evaluate blood flow over 1-2 minutes. In cases of partial or no effect, repeat fibrinolytic agent injection at the same dose. 4) In cases of fibrinolytic agent ineffectiveness, perform revascularization of the distal parts of the thrombosed branch with the superficial temporal artery or arteries adjacent to the MCA.

\section{Conclusions}

An IAT was observed in $18.9 \%$ of the 53 patients who underwent surgical treatment for partially thrombosed MCA aneurysms. Thrombectomy with temporary trapping was the major risk factor for this complication.

Puncture of the wall of the thrombosed MCA branch and intraarterial injection of a fibrinolytic agent is one possible solution of this problem and has proven highly effective. When fibrinolysis fails to restore blood flow, revascularization of the distal portions of the thrombosed arteries should be performed.

\section{References}

1. Credo RB, Burke SE: Fibrinolytic mechanism, biochemistry, and preclinical pharmacology of recombinant prourokinase. J Vasc Interv Radiol 6 (6 Pt 2 Suppl):8S-18S, 1995

2. Drake CG: Giant intracranial aneurysms: experience with surgical treatment in 174 patients. Clin Neurosurg 26:12-95, 1979

3. Dujovny M, Osgood CP, Barrionuevo PJ, Perlin A, Kossovsky N: SEM evaluation of endothelial damage following temporary middle cerebral artery occlusion in dogs. J Neurosurg 48:42-48, 1978

4. Furlan A, Higashida R, Wechsler L, Gent M, Rowley H, Kase $\mathrm{C}$, et al: Intra-arterial prourokinase for acute ischemic stroke. The PROACT II study: a randomized controlled trial. JAMA 282:2003-2011, 1999

5. Karpenko AA, Starosotskaya MV, Chernyavsky MA, Chernyavsky AM, Belogurov AA, Del'ver EP: Thrombolytic therapy of acute massive thromboembolism of the pulmonary artery with the use of recombinant prourokinase. Phlebologiya $4: 16-20,2010$

6. Kato Y, Sano H, Imizu S, Yoneda M, Viral M, Nagata J, et al: Surgical strategies for treatment of giant or large intracranial aneurysms: our experience with 139 cases. Minim Invasive Neurosurg 46:339-343, 2003 
7. Krylov VV, Burov SA, Dash'ian VG, Galankina IE: [Local fibrinolysis in the surgery of non-traumatic intracranial hemorrhages.] Vestn Ross Akad Med Nauk 2013:24-31, 2013 (Russian)

8. Krylov VV, Klimov AB, Polunina NA: [Morphological, clinical, diagnostic features and the treatment of patients with giant brain aneurisms.] Zh Nevrol Psikhiatr Im S S Korsakova 111:15-22, 2011 (Russian)

9. Kühnel TS, Müller GH: Experimental animal studies of clipinduced microvascular trauma. Microsurgery 24:241-247, 2004

10. Lavine SD, Masri LS, Levy ML, Giannotta SL: Temporary occlusion of the middle cerebral artery in intracranial aneurysm surgery: time limitation and advantage of brain protection. J Neurosurg 87:817-824, 1997

11. Lawton MT, Quiñones-Hinojosa A, Chang EF, Yu T: Thrombotic intracranial aneurysms: classification scheme and management strategies in 68 patients. Neurosurgery 56:441-454, 2005

12. O'Neill M, Hope T, Thomson G: Giant intracranial aneurysms: diagnosis with special reference to computerised tomography. Clin Radiol 31:27-39, 1980

13. Ouriel K, Kandarpa K, Schuerr DM, Hultquist M, Hodkinson G, Wallin B: Prourokinase versus urokinase for recanalization of peripheral occlusions, safety and efficacy: the PURPOSE trial. J Vasc Interv Radiol 10:1083-1091, 1999

14. Pilipenko IuV, Éliava ShSh, Iakovlev SB, Belousova OB, Buklina SB, Arustomian SR, et al: [The analysis of complications of surgical treatment of brain aneurysms in patients operated on in the late posthemorrhagic period.] Vopr Neirokhir 78:32-39, 2014 (Russian)

15. Pilipenko IuV, Éliava ShSh, Shekhtman OD, Kheřreddin AS: [Local fibrinolysis in treatment of non-traumatic intracerebral hematomas and ventricular hemorrhages]. Vopr Neirokhir 76:3-13, 2012 (Russian)

16. Rodríguez-Hernández A, Sughrue ME, Akhavan S, Habdank-Kolaczkowski J, Lawton MT: Current management of middle cerebral artery aneurysms: surgical results with a "clip first" policy. Neurosurgery 72:415-427, 2013

17. Sano H, Kato Y, Shankar K, Kanaoka N, Hayakawa M, Katada K, et al: Treatment and results of partially thrombosed giant aneurysms. Neurol Med Chir (Tokyo) 38 Suppl:58-61, 1998
18. Saribekian AS, Arzamastsev EV, Bibilashvili RSh, Golubykh VL, Belogurov AA, Poliakova LN, et al: [Local fibrinolysis of hypertensive intracerebral hematomas.] Vopr Neirokhir 3:14-20, 2008 (Russian)

19. Shi ZS, Ziegler J, Duckwiler GR, Jahan R, Frazee J, Ausman JI, et al: Management of giant middle cerebral artery aneurysms with incorporated branches: partial endovascular coiling or combined extracranial-intracranial bypass - a team approach. Neurosurgery 65 (6 Suppl):121-131, 2009

20. Trukhanova IG: [The use of recombinant urokinase for prehospital thrombolysis in patients with acute coronary syndrome.] Klin Pharmakol Ter 19:60-65, 2010 (Russian)

21. Uede T, Ohtaki M, Tanabe S, Hashi K: [Direct surgical management of giant and large intracerebral aneurysms, associated with intraluminal thrombus and/or atherosclerotic thickening of aneurysmal neck.] No Shinkei Geka 25:1007-1015, 1997 (Jpn)

22. Whittle IR, Dorsch NW, Besser M: Spontaneous thrombosis in giant intracranial aneurysms. J Neurol Neurosurg Psychiatry 45:1040-1047, 1982

\section{Disclosure}

The authors report no conflict of interest concerning the materials or methods used in this study or the findings specified in this paper.

\section{Author Contributions}

Conception and design: Pilipenko, Shekhtman. Acquisition of data: Pilipenko, Konovalov. Analysis and interpretation of data: Pilipenko. Drafting the article: Pilipenko. Critically revising the article: Eliava. Statistical analysis: Pilipenko. Administrative/ technical/material support: Eliava. Study supervision: Eliava. Translation of text: Shekhtman.

\section{Correspondence}

Yury Pilipenko, Vascular (3rd) Department, Burdenko Neurosurgical Institute, 4th Tverskaya-Yamskaya St., Office 615 , Moscow 125047, Russia. email: coronainfo@gmail.com. 